\title{
STRUCTURE ANALYSES COMBINING ELECTRON DIFFRACTION AND MICROWAVE DATA
}

\section{STUDY OF $\mathrm{XeOF}_{4} *$}

\author{
E. JEAN JACOB, H. BRADFORD THOMPSON** AND L. S. BARTELL*** \\ Department of Chemistry, University of Michigan, Ann Arbor, Mich. 48104 (U.S.A.)
}

(Received March 9th, 1970; in revised form August 10th, 1970)

\section{ABSTRACT}

Molecular parameters for $\mathrm{XeOF}_{4}$ derived from electron diffraction data are compared with those previously reported in vibrational and rotational spectroscopic studies of the molecule. Additional least-squares calculations were performed which simultaneously fitted the diffraction and microwave data. Although results for the separate experiments are in good agreement, each set of parameters suffers from fairly large uncertainties which are largely unrelated to the accuracy of the respective physical measurements. A comparison of parameter correlations for the separate and joint least-squares calculations indicates that the troublesome correlations in the individual sets of data are significantly reduced when the two sets of data are combined. Various interpretational difficulties associated with the process of combining the data from the two experiments are discussed and suggestions are made for estimating uncertainties in cases where the observational residuals are far from random.

\section{INTRODUCTION}

In principle it is possible, through appropriate transformations, to reduce electron diffraction and microwave data to a common basis for comparison, such as the $r_{z}$ or zero point average structure ${ }^{\mathbf{x}}$. In recent years, Kuchitsu and coworkers have explored various aspects of comparisons between $r_{z}$ parameters derived from the two experiments separately and problems associated with combining dissimilar data to obtain a single set of optimized structure parameters ${ }^{2}$. For many molecules,

* This research was supported by a grant from the National Science Foundation. Results were presented in part, at the Symposium on Molecular Structure and Spectroscopy, Columbus, Ohio, September 8, 1967.

** Present address: Department of Chemistry, University of Toledo, Toledo, Ohio, U.S.A.

*** Author to whom correspondence concerning reprints should be addressed. 
however, the analyses required for rigorous transformation of spectroscopic and diffraction data to an $r_{\mathbf{z}}$ basis either are not feasible or suffer from large uncertainty or indeterminacy. In cases where the combination of electron diffraction and microwave data must be made at some cruder level of approximation, it is pertinent to investigate the potential benefits associated with combining the data.

Xenon oxide tetrafluoride appeared to be a promising example for study. Several years ago Martins and Wilson ${ }^{3}$ reported rotational constants for enough isotopic species to allow the calculation of all geometric parameters ${ }^{4}$. Electron diffraction is presumably capable of establishing the parameters also, but with a different pattern of sensitivity to experimental and interpretational uncertainties. The largest spectroscopic problem was the uncertainty in the position of the xenon (lying near the center of mass) and the attendant strong correlation between the derived $\mathrm{XeO}$ bond length and $\mathrm{O}-\mathrm{Xe}-\mathrm{F}$ bond angle. The principal difficulty anticipated for the diffraction analysis, on the other hand, was the proper resolution of the $\mathrm{XeO}$ and $\mathrm{XeF}$ bond lengths from each other in the face of the distortions of peak shapes known to arise in the case of bonds linking light and heavy atoms. One aspect of composite least-squares calculations that has not been discussed in previous studies is the variation in parameter correlations with different combinations of data. We found for $\mathrm{XeOF}_{4}$ that these changes were the most direct indicators of the advantages of combining the data.

Xenon oxide tetrafluoride is of interest in connection with current models for directed valence, since it can be viewed as having a Xe-O double bond competing with an opposed lone pair for space on the xenon coordination sphere. Comparisons of $\mathrm{XeOF}_{4}$ with related iodine and xenon fluorides and oxyfluorides which address this problem have been made elsewhere ${ }^{5}$.

\section{EXPERIMENTAL PROCEDURE}

Argonne National Laboratory supplied the sample of $\mathrm{XeOF}_{4}$ used to obtain diffraction patterns. An input system of nickel, Monel metal, and stainless steel was used, all surfaces in contact with the sample being preseasoned with $\mathrm{ClF}_{3}$. Typical exposure times were 1-5 sec, and absorbances ranged from 0.2 to 1.5 . Patterns were recorded on Kodak $4 \times 5$ in. process plates at the $21-\mathrm{cm}$ camera distance using both an $R^{2}$ and an $R^{3}$ rotating sector, and at the $11-\mathrm{cm}$ and $7-\mathrm{cm}$ camera distances using the $R^{3}$ sector. Standard procedures were used to obtain absorbances from the photographic plates.

\section{PRELIMINARY TREATMENT OF DATA}

Experimental levelled intensities, $I_{0}(s)$, as defined elsewhere ${ }^{6,7}$ were obtained by averaging data from several plates at each of the camera distances. 
Calculated atomic and reduced molecular intensities were based on partial wave elastic scattering factors of Cox and Bonham ${ }^{8}$, Tavard's inelastic scattering factors for oxygen and fluorine ${ }^{9}$, and xenon inelastic scattering factors obtained by scaling the Pohler and Hanson values for iodine ${ }^{10}$ according to the Heisenberg expression for $S(s)$ as a function of atomic number ${ }^{11}$.

PROCEDURES FOR REFINEMENT OF MOLECULAR PARAMETERS

Molecular parameters were refined by comparing calculated and experimental reduced molecular intensities, $M(s)$, where

$$
M(s)=\left[I_{\mathrm{o}}(s) / I_{\mathrm{B}}(s)\right]-1,
$$

by a least-squares process. Initially, data for the individual camera distances were treated separately. After the background functions, $I_{\mathrm{B}}(s)$, for the separate camera distances had been refined, using the usual criteria, a blended experimental $M(s)$ curve was constructed, merging data from the $21-\mathrm{cm}$ and $11-\mathrm{cm}$ camera distances over the range $s=5.3-38.9 \AA^{-1}$. The original data for $I_{0}(s)$ and $I_{\mathrm{B}}(s)$ are available from ASIS*. Data below $s=5.3 \AA^{-1}$ were discarded because of the high sensitivity of derived parameters to the relatively large failures in scattering theory at small scattering angles. The 7-cm data were discarded also because they appeared to be of substantially lower quality than the other data. Fig. 1 shows the experimental $s M(s)$ together with a typical calculated $s M(s)$ curve and the corresponding difference curve.

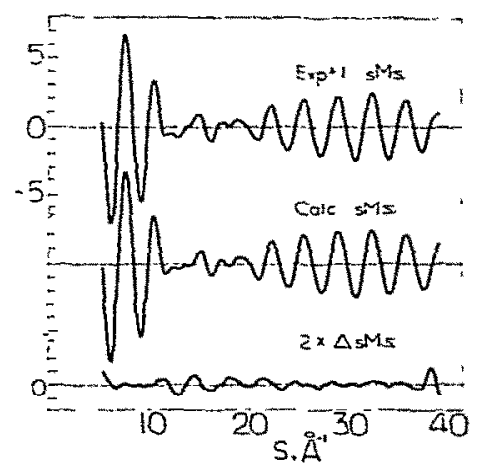

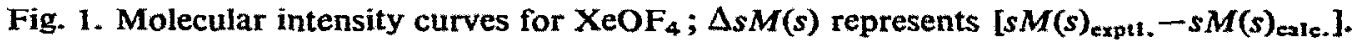

Asymmetry constants of $2.0 \AA^{-1}$ and $1.0 \AA^{-1}$ were assumed for the bonded and nonbonded distances respectively. For least-squares refinements, which imposed geometrical self-consistency on the internuclear distances, the nonbonded

* For a listing of experimental intensity data order document NAPS-01138 from ASIS-National Auxiliary Publications Service, c/o CCM Information Corporation, 909 Third Avenue, New York, N.Y., 10022 ; remitting $\$ 2.00$ for each microfiche or $\$ 5.00$ for each photocopy. 
distances were corrected for Bastiansen-Morino shrinkage effects. Estimates of $0.001 \AA, 0.002 \AA$, and $0.007 \AA$ for the OF, FF, and F . F F shrinkages* were based on calculated shrinkages for $\mathrm{XeF}_{4}{ }^{12}$ and $\mathrm{TeF}_{6}{ }^{13}$ and are subject to large relative uncertainties ${ }^{14}$. Poor resolution of the OF and FF distances made it impracticable to refine separate vibrational amplitudes for these two distances. A constraint of the form $l_{\mathrm{FF}}-l_{\mathrm{OF}}=\Delta$, where $\Delta$ was fixed for any particular calculation, was imposed in the results reported below.

Experimental radial distribution curves

$$
f(r)=\int s M(s) \mathrm{e}^{-b s^{2}} \sin s r \mathrm{~d} s
$$

were calculated using the $M_{n}(s)$ approximation ${ }^{7}$ and a value for $b$ of $0.0015 \AA^{2}$. Fig. 2 shows the experimental $f(r)$ calculated from the $s M(s)$ curve of Fig. 1.

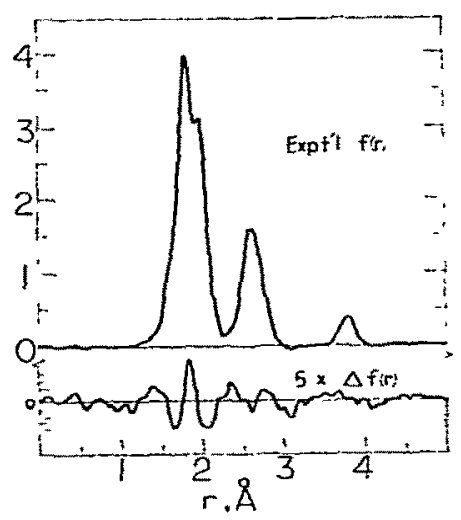

Fig. 2. Radial distribution function for $\mathrm{XeOF}_{4} ; \Delta f(r)$ represents $\left[f(r)_{\text {exptl. }}-f(r)_{\text {calc. }}\right]$.

Incorporation of microwave data into some of the least-squares refinements was accomplished in the following manner. Additional observational equations were generated using as data the observed effective moments of inertia, $I_{b}$, for several isotopic species, and the distance of the oxygen atom from the center of mass of the ${ }^{129} \mathrm{Xe}^{16} \mathrm{OF}_{4}$ species, $z_{\mathrm{ox}}$, derived from the appropriate $I_{b}$ 's. Thus the quantity being minimized with respect to a set of parameters, $\left\{\theta_{i}\right\}$, was

$$
S\left(\left\{\theta_{i}\right\}\right)=\sum_{\substack{\text { Intensity } \\ \text { points }}} w_{j} \delta_{j}^{2}\left[s I_{0}(s)\right]+\sum_{\substack{\text { Moments } \\ \text { of inertia }}} w_{k} \delta_{k}^{2}\left(I_{b}\right)+w_{m} \delta^{2}\left(z_{o x}\right)
$$

where $\delta$ denotes the difference between observed and calculated values of the indicated quantities. Selection of weights, $w$, is discussed in subsequent sections. The parameters, $\left\{\theta_{i}\right\}$, were on an $r_{\mathrm{g}}$ basis appropriate for the diffraction data. Expressions for calculating $I_{b}$ and $z_{\mathrm{ox}}$ were based on simplified, non-rigorous transformations between microwave parameters and these $\theta$ 's as outlined below. First,

* Throughout this paper, FF denotes the short and F ... F the long fluorine-fluorine distances. 
$I_{b}$ and $z_{0 x}$ were both written as functions of the same type of internuclear distance, denoted by $r_{M w}$ below. Secondly, the same "shrinkage" parameter, $\varepsilon$, was used to relate each $\boldsymbol{r}_{\mathrm{g}}$ (for bonds) to its corresponding $r_{\mathrm{MW}}$. Finally, it was assumed that a working value for $\varepsilon$ might be estimated from the difference between $r_{\mathrm{g}}$ and $r_{\mathrm{z}}$, a quantity which can be calculated from mean square perpendicular amplitudes or estimated from known corrections for other molecules. This last assumption is partially justified by the observation that, for the molecules for which $r_{g}, r_{z}$, and $r_{0}$ have been determined, $r_{z}$ is usually substantially closer to $r_{0}$ than to $r_{g}$. From calculated mean square perpendicular amplitudes for $\mathrm{XeOF}_{4}{ }^{15}$ and $\mathrm{TeF}_{6}{ }^{16}$, we arrived at a value for $\varepsilon$ of $0.003 \AA$. Least-squares calculations using only microwave data were made subject to the first of the above restrictions.

\section{RESULTS}

\section{From diffraction data alone}

All of our calculations were based on the $C_{4 v}$ symmetry for $\mathrm{XeOF}_{4}$ indicated by vibrational and rotational spectroscopy $3,4,17$. By inspection, neither the $C_{2 \mathrm{v}}$ or $C_{3 v}$ trigonal bipyramidal structures are compatible with the diffraction data.

From the five distinct internuclear distances contributing to the molecular intensity, three structural parameters must be derived, a natural set being the $\mathrm{XeO}$ and $\mathrm{XeF}$ bond lengths, denoted as $r_{\mathrm{XcO}}$ and $\boldsymbol{r}_{\mathrm{XcF}}$ below, and the $\mathrm{O}-\mathrm{Xe}-\mathrm{F}$ angle, denoted as $\alpha$. Comparisons of geometrically consistent least-squares refinements with those which allowed each internuclear distance to vary independently reveals that the two bond distances are determined almost entirely from the scattering from the bonds themselves, and not from the interdependence of bonded and nonbonded distances required for geometrical self-consistency. Calculations based solely on random error theory indicate a large correlation between $r_{\mathrm{XeO}}$ and $r_{\mathrm{XeF}}$, and modest correlations between $\alpha$ and either bond length (see Table 2A).

However, the angle $\alpha$ is correlated strongly with amplitudes of vibration of the $\mathrm{OF}$ and $\mathrm{FF}$ distances and consequently with the value of $\Delta$ (see preceding section) used in calculations. Evidence from other molecules suggests that deficiencies in scattering theory for bonds containing atoms of widely differing atomic numbers affect regions of $r$-space on either side of the bond in question. For this reason, simple optimization of the fit to the geminal non-bonded peak, which yields a value of $0.025 \AA$ for $\Delta$, is subject to considerable uncertainty. We chose to use a larger value, $0.060 \AA$, in accord both with various spectroscopic calculations for $\mathrm{XeF}_{4}$ and $\mathrm{XeOF}_{4}{ }^{14,15,18}$ and with the misfit expected in the affected region of $r$-space on the basis of diffraction data for $\operatorname{ReF}_{6}{ }^{19}$, a simple octahedral molecule with a heavy central atom. Although this choice resulted in a significant increase in $\sigma\left[I_{0}(s)\right]$, only slight increases in parameter standard errors were observed. A 
TABLE 1

MOLECULAR PARAMETERS FOR XENON OXIDE TETRAFLUORIDE ${ }^{2}$

\begin{tabular}{|c|c|c|c|c|}
\hline $\begin{array}{l}\text { Parameterldata } \\
\text { fitted }\end{array}$ & $\begin{array}{l}A^{\mathrm{b}} \\
I_{o}(s)\end{array}$ & $\begin{array}{l}B^{c} \\
\left\{I_{0}(s), I_{b}, z_{o x}\right\}\end{array}$ & $\begin{array}{l}C^{\mathrm{d}} \\
\left\{I_{b}, z_{a x}\right\}\end{array}$ & $\begin{array}{l}D^{\mathrm{e}} \\
\text { Spectroscopic }\end{array}$ \\
\hline$r_{g}(\mathrm{XeO}), \AA$ & $1.7109 \pm 0.011(0.0020)$ & $1.7081 \pm 0.007(0.0017)$ & $1.7053(0.00089)$ & $1.706 \pm 0.015$ \\
\hline$r_{\varepsilon}(\mathrm{XeF}), \AA$ & $1.9010 \pm 0.0032(0.00079)$ & $1.9023 \pm 0.0023(0.00062)$ & $1.9040(0.00002)$ & $1.903 \pm 0.005$ \\
\hline$\Varangle O-X e-F$, degrees & $91.93 \pm 0.66(0.19)$ & $91.62 \pm 0.23(0.14)$ & $91.66(0.072)$ & $91.8 \pm 0.5$ \\
\hline$l_{\mathrm{z}}(\mathrm{XeO}), \AA$ & $0.0364 \pm 0.011(0.0021)$ & $0.0369(0.0020)$ & -- & 0.0362 \\
\hline$l_{g}(\mathrm{XeF}), \AA$ & $0.0484 \pm 0.0034(0.00078)$ & $0.0485(0.00075)$ & - & 0.0446 \\
\hline$I_{g}(\mathrm{OF}), \AA$ & $0.0740 \pm 0.010(0.0029)$ & $0.0729(0.0028)$ & -- & 0.0803 \\
\hline$l_{z}(F F), \AA^{s}$ & {$[0.1355] \pm 0.010$} & {$[0.1343]$} & -- & 0.1203 \\
\hline$l_{z}(F \cdots F), \AA$ & $0.0670 \pm 0.0035(0.0078)$ & $0.0672(0.0077)$ & -- & 0.0610 \\
\hline$\sigma\left[I_{0}(s)\right] /{\overline{I_{0}(s)^{2}}}^{2}$ & 0.000740 & 0.000763 & 0.000810 & - \\
\hline$\sigma\left(I_{b}\right)$, a.m.u. $\AA^{2^{b, 1}}$ & 0.057 & 0.120 & 0.0009 & 0.015 \\
\hline$z_{0 x}, \hat{A}^{b .3}$ & 1.6062 & 1.6002 & 1.5980 & 1.6002 \\
\hline
\end{tabular}

" Uncertainties prefixed by " \pm " include contributions from both systematic and randorn errors. For the electron diffraction data, they are estimated standard errors. For the microwave results, they are of unspecified statistical significance. For the composite data, they are estimated as described in the text. Values enclosed in parentheses are based on random error theory only and are chiefiy measures of the internal consistency of the data being fitted.

${ }^{b}$ Least-squares fit to $I_{0}(s)$ only, using weights proportional to $s^{2} \cdot \sigma\left(I_{b}\right)$ and $z_{o v}$ were evaluated on the basis of $r_{\mathrm{MW}}=r_{\mathrm{z}}-0.003 \AA$.

c Least-squares fit of $I_{0}(s)$, seven moments of inertia, and $z_{0 x}$. Weights for $I_{0}(s)$ were proportional to $s^{2}$. Each $I_{b}$ was taken to be equivalent to one observation and $z_{\mathrm{ax}}$ equivalent to three observations.

d Least-squares fit of seven $I_{b}$ 's and $z_{\mathrm{ox}}$, using weight $\left(I_{b}\right)$ per observation $=0.0014 \times$ weight $\left(z_{0 x}\right)$, with each quantity used as a single observation. Derived bond lengths were augmented by $0.003 \AA$ to put them on an $r_{z}$ basis for representation in the table.

- Structural parameters are from Martins and Wilson (see ref. 4), the r's having been augmented by $0.003 \AA$ to put them on an $r_{z}$ basis. The amplitudes of vibration are those of Cyvin, Brunvoll and Robiette (see ref. 14). I $l_{\alpha}(\mathrm{FF})$ constrained to be equal to $l_{\alpha}(\mathrm{OF})+0.06 \AA$ (see text). Resultant $l_{g}$ 's are reported in the table.

= For column $\mathbf{C}$ this was calculated for the given geometry, using amplitudes of vibration as in column $\mathbf{A}$, and docs not correspond to a least-squares refinement based on $I_{0}(s)$.

"For column $A,\left(I_{b}\right)$ and $z_{a x}$ were calculated using $r_{M w}$ 's derived from the reported $r_{g}$ 's as described in the text. For column D, Wilson's uncorrected parameters were used.

' For the ${ }^{129} \mathrm{Xe}^{16} \mathrm{OF}_{4}$ species on the ${ }^{12} \mathrm{C}$ mass scale, the experimental $I_{b}=181.376$ a.m.u. $\AA^{2}$.

${ }^{3}$ For the ${ }^{129} \mathrm{Xe}^{16} \mathrm{OF}_{4}$ center-of-mass coordinate system. Using experimental $I_{b}\left({ }^{129} \mathrm{Xe}^{16} \mathrm{OF}_{4}\right)$ and $I_{b}\left({ }^{129} \mathrm{Xe}^{18} \mathrm{OF}_{4}\right)$ in Kraitchman's equation, we calculated $z_{\mathrm{ex}}$ to be $1.598 \AA$.

change in $\Delta$ from $0.025 \AA$ to $0.060 \AA$ carries $l_{\mathrm{FF}}$ from $0.100 \AA$ to $0.134 \AA$ and brings about a decrease in $\alpha$ of $1^{\circ}$. Error estimates for $\alpha$ given in Table 1 take into account the uncertainty in $\Delta$.

Column A of Table 1 gives results of the refinement of electron diffraction intensities only. Two standard deviations are given for each parameter. One is calculated assuming randomly distributed least-squares residuals and optimum weighting of uncorrelated observations. The other represents an estimate of overall 
uncertainty, including systematic errors arising both from the experiment and from deficiencies in the scattering theory, as well as adjustments for the correlation between intensity points ${ }^{6}$. While the intensity data contain all of the information available on systematic deviations between experiment and theory, the localized distortions in the vicinity of the heavy atom-light atom peaks make the $\Delta f(r)$ curve of Fig. 2 a simpler function to diagnose. The systematic effects are also manifested in the observed correlation between the least-squares weight function and the derived bond lengths, the bond lengths decreasing if the data for small scattering angles are weighted very heavily*. Although it is, as yet, impossible to transform rigorously from observed non-random distributions of residuals in heavy-atom molecules into corresponding parameter errors, we feel we can make error estimates for those parameters most seriously affected which will be superior to the usual estimates obtained by arbitrarily augmenting the "standard errors" derived blindly from intensity residuals. Our approach is to estimate the possible influence of the distortions in the $f(r)$ curve upon the derived structure parameters by the expres$\operatorname{sion}^{6}$

$$
\sigma\left(r_{k}\right)=\left(\frac{n}{n-m}\right)^{\frac{1}{2}}\left(32 b L^{2}\right)^{\frac{1}{2}}\left(\frac{L^{2}}{L^{2}+2 b}\right)^{\frac{2}{2}} \frac{\sigma[f(r)]}{\left(f_{\max }\right)_{k}}
$$

where $L^{2}=l^{2}+2 b$, and the error level $\sigma[f(r)]$ in the immediate region of the radial distribution peaks in question is treated as if it arose from random noise. Although the expression is valid only for truly statistical errors, for well-resolved peaks, and for modest failure of the Born approximation, it takes into account much more directly than do conventional analyses on intensities those nonrandom errors which are delocalized in intensities but localized in $f(r)$. The uncertainty associated with $\alpha$ was derived from the resultant $\sigma\left(r_{i j}\right)$ values and the geometrical expression connecting them. The $\sigma\left(r_{\mathrm{OF}}\right)$ used also included the uncertainty in the least-squares fitting of the intensities and the concomitant uncertainty in $\Delta$.

Systematic errors which are concentrated in certain regions of $r$-space can shift vibrational amplitudes as well as internuclear distances, and by comparable amounts. Our reported uncertainties for $l_{\mathrm{XeO}}$ and $l_{\mathrm{XeF}}$ are based on the $\sigma\left(r_{\mathrm{XeO}}\right)$ and $\sigma\left(r_{\mathrm{XeF}}\right)$ estimated as indicated above. Values for $\sigma\left(l_{\mathrm{OF}}\right)$ and $\sigma\left(l_{\mathrm{FF}}\right)$ were calculated assuming an uncertainty in $\Delta$ of $0.02 \mathrm{~A}$ and ignoring the correlation between $\Delta$ and the mean of $l_{\mathrm{OF}}$ and $l_{\mathrm{FF}}$. No attempt was made to estimate the uncertainty in $l_{i j}$ 's introduced by uncertainties in the phase shift envelopes, $\cos \left(\Delta \eta_{i j}\right)$.

\section{From microwave data alone}

Column D of Table 1 reproduces the parameters and uncertainties reported by Martins and Wilson ${ }^{4}, \varepsilon=0.003 \AA$, having been added to $r_{\mathrm{XeO}}$ and $r_{\mathrm{XeF}}$ for

\footnotetext{
* This feature is not unique to $\mathrm{XeOF}_{4}$, but has been observed for all molecules with heavy atoms
} studied in this laboratory, including several iodine, xenon, and rhenium compounds. 
TABLE 2

DIAGONAL ELEMENTS AND CORRELATION COEFFICIENTS FOR MOLECULAR PARAMETERS OF XeOF 4 " A. Complete matrix for least-squares refinement on $I_{0}(s)^{b}$

\begin{tabular}{|c|c|c|c|c|c|c|c|c|c|}
\hline & $r_{X=0}$ & $r_{\text {XeF }}$ & $\Varangle O-X e-F$ & $l_{x \in O}$ & $l_{X \in F}$ & $l_{\text {OF }}$ & $I_{F F}$ & $I_{\boldsymbol{F}} \ldots \boldsymbol{F}$ & $\boldsymbol{R}$ \\
\hline $\begin{array}{l}\sigma\left(\theta_{l}\right) \\
r_{\text {XeO }} \\
r_{\text {XeF }} \\
\Varangle \mathbf{O}-X e-F\end{array}$ & $\begin{array}{l}0.0020 \\
1\end{array}$ & $\begin{array}{c}0.00079 \\
-0.52 \\
1\end{array}$ & $\begin{array}{r}0.19 \\
-0.13 \\
-0.10 \\
1\end{array}$ & 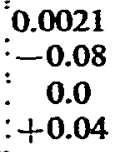 & $\begin{array}{c}0.00078 \\
+0.03 \\
+0.03 \\
+0.04\end{array}$ & $\begin{array}{l}0.0029 \\
-0.02 \\
+0.01 \\
-0.34\end{array}$ & $\begin{array}{c}0.0029 \\
0.0 \\
-0.05 \\
+0.75\end{array}$ & $\begin{array}{c}0.0078 \\
0.0 \\
0.0 \\
+0.03\end{array}$ & $\begin{array}{r}0.013 \\
-0.13 \\
+0.13 \\
+0.14\end{array}$ \\
\hline $\begin{array}{l}l_{\text {XeO }} \\
I_{\text {XcF }} \\
l_{\text {OF }} \\
l_{F F} \\
l_{F} \ldots F \\
R, \text { index of }\end{array}$ & f resolut & & & 1 & $\begin{array}{c}-0.52 \\
1\end{array}$ & $\begin{array}{c}0.0 \\
+0.07 \\
1\end{array}$ & $\begin{array}{c}+0.03 \\
+0.16 \\
-0.40 \\
1\end{array}$ & $\begin{array}{c}+0.01 \\
+0.04 \\
+0.01 \\
+0.04 \\
1\end{array}$ & $\begin{array}{l}+0.07 \\
+0.49 \\
+0.11 \\
+0.35 \\
+0.09 \\
1\end{array}$ \\
\hline
\end{tabular}

B. Fitting $I_{0}(s), I_{b}, z_{o x}$ simultaneouslyc

\begin{tabular}{llll}
\hline & $r_{\text {XeO }}$ & $r_{\text {XcF }}$ & $\Varangle O-X e-F$ \\
\hline$\sigma\left(\theta_{l}\right)$ & 0.0017 & 0.00062 & 0.14 \\
$r_{\text {XeO }}$ & 1 & -0.40 & -0.73 \\
$r_{\text {XeF }}$ & & 1 & +0.07 \\
$\Varangle O-X e-F$ & & & 1 \\
\hline
\end{tabular}

C. Fitting microwave data only, with small ${ }^{\mathrm{d}} w t\left(I_{b}\right) / w t\left(z_{v x}\right)$

\begin{tabular}{llll}
\hline & $r_{\text {XeO }}$ & $r_{\text {XeF }}$ & $\Varangle 0-X e-F$ \\
\hline$\sigma\left(\theta_{i}\right)$ & 0.00089 & 0.00002 & 0.072 \\
$r_{\text {XeO }}$ & 1 & +0.58 & -0.999 \\
$r_{\text {XeF }}$ & & 1 & -0.62 \\
$\Varangle O-X e-F$ & & & 1 \\
\hline
\end{tabular}

D. Fitting microwave data only, with large ${ }^{\mathrm{c}} w t\left(I_{b}\right) / w t\left(z_{0 x}\right)$

\begin{tabular}{llll}
\hline & $r_{\text {XeO }}$ & $r_{\text {XeF }}$ & $\Varangle 0-X e-F$ \\
\hline$\sigma\left(\theta_{t}\right)$ & 0.00053 & 0.00003 & 0.041 \\
$r_{\text {XeO }}$ & 1 & -0.13 & -0.99 \\
$r_{\text {XeF }}$ & & 1 & -0.03 \\
$\Varangle O-X e-F$ & & & 1 \\
\hline
\end{tabular}

Coefficients are taken directly from $B^{-1}$ matrices:

$$
\rho_{i j}=B_{i j}{ }^{-1} /\left(B_{i l^{-1}} \times B_{J j}{ }^{-1}\right)^{\frac{1}{2}} \quad \sigma\left(\theta_{l}\right)=\left(B_{i l}{ }^{-1}\right)^{\frac{1}{2}}\left[\frac{V^{\prime} \mathbf{W V}}{n-m}\right]^{\frac{1}{3}}
$$

- Corresponds to column A of Table 1 . $l_{\text {FF }}-l_{\text {or }}$ constrained to be $0.060 \AA$ during least-squares refinement.

- Corresponds to column B of Table 1 , for which $w t\left(I_{b}\right) / w t\left(z_{o x}\right)=4.36 \times 10^{-5}$. Ideally this ratio should be the same as $\sigma^{2}\left(z_{o x}\right) / \sigma^{2}\left(I_{b}\right)$, which from Table 1 has the value $3.36 \times 10^{-4}$ if $\sigma\left(z_{o x}\right)$ is taken to be $\left\{z_{\mathrm{ox}}\right.$ (obs.) $-z_{\mathrm{ox}}$ (calc.) $\}$. Correlations between other parameters are only slightly different from those obtained fitting $I_{0}(s)$ only. See subtable A.

${ }^{d}$ This corresponds to column $C$ of Table 1 , for which $w t\left(I_{b}\right) / w t\left(z_{o x}\right)=1.4 \times 10^{-3}$ and $\sigma^{2}\left(z_{o x}\right) /$ $\sigma^{2}\left(I_{b}\right)=0.61 \times 10^{-3}$.

e This corresponds to $\mathrm{wt}\left(I_{b}\right) / \mathrm{wt}\left(z_{\mathrm{ox}}\right)=0.10, \sigma\left(I_{b}\right)=3.73 \times 10^{-4}$ a.m.u. $\AA^{2}, z_{\mathrm{ox}}$ (obs.) $-z_{\mathrm{ox}}$ (calc.) $=0.00025 \AA$, and $\sigma^{2}\left(z_{\mathrm{ox}}\right) / \sigma^{2}\left(I_{b}\right)=0.46$. 
purposes of comparison with other columns of the table, which are all on an $r_{\mathrm{g}}$ basis. The uncertainties include at least a rough estimate of systematic errors associated with problems peculiar to extraction of structural parameters from ground state rotational constants and with the substitution method. Comparison of columns $A$ and $D$ shows the electron diffraction and microwave parameters to be the same within estimated uncertainties, even without allowing an uncertainty of $0.002-0.003 \AA$ in the adjustment of the parameters of the two experiments to a common basis. This is gratifying in view of the unsettled state of electron scattering theory for heavy atoms.

Least-squares calculations using only $z_{0 x}$ and the seven $I_{b}$ 's as data were made with a range of relative weights for $z_{\mathrm{ox}}$ and $I_{b}$. Column $C$ of Table 1 gives results for one such calculation. The very small "standard deviations" simply reflect the fact that adjustment of three parameters to fit the eight observables results in very small residuals, especially since the seven $I_{b}$ 's all embody nearly the same systematic biases. Because the residuals cannot be taken as random, and because the weighting scheme did not take into account the fact that the $I_{b}$ 's are at best marginally independent observations, no physical significance can be attached to the computed standard deviations.

Although values for the parameters are insensitive to the relative weights for $I_{b}$ and $z_{\mathrm{ox}}$ (over the entire range of ratios 0 to $\infty$ ), some of the coefficients of the correlation matrices were highly dependent on the relative weights, as illustrated in Tables 2C and 2D. Increasing the weight for $z_{\mathrm{ox}}$ has the effect of coupling the two bond lengths and of correlating the bond angle, $\alpha$, with the $\mathrm{XeF}$ bond. Over the entire range of weights the correlation coefficient between $r_{\mathrm{XeO}}$ and $\alpha$ remains very high, ranging between -0.985 and -1.0 .

\section{Calculations combining diffraction and spectroscopic data}

As pointed out by Kuchitsu ${ }^{2}$, in the presence of systematic errors, the conventional inverse proportionality between weights and the corresponding standard deviations of observations does not give an adequate basis for assigning weights in least-squares calculations combining different types of observations. It seems reasonable to select relative weights consistent with the interpretational uncertainties of the observations if these are larger than the direct errors in measurement. Using the $\sigma\left[s I_{0}(s)\right]$ determined from least-squares fits, together with estimated values for $\sigma\left(z_{\mathrm{ox}}\right)$ and $\sigma\left(I_{b}\right)$ of $0.002 \AA$ and 0.3 a.m.u. $\AA^{2}$ respectively*, we obtained, from the standard least-squares formula, weights for $z_{o x}$ and $I_{b}$ of $6.6 \times 10^{3}$ and 0.29 respectively for $s I_{0}(s)$ values of unit weight. We retained these weights for single "observations" and merely adjusted the number of observations to which each microwave datum was considered to be equivalent. Column B of Table 1 gives

* For a change in $r_{\mathrm{XeF}}$ of $0.002 \AA, I_{b}$ changes by 0.3 a.m.u. $\AA^{2}$. 
results for a calculation which used seven moments of inertia, each weighted as one observation, plus $z_{\text {ox }}$, weighted as three observations, in addition to $108 s I_{0}(s)$ values of unit weight. Acceptable values for $\sigma\left(I_{b}\right)$ and $\delta z_{o x}$ (obs.-calc.) were obtainable for a fairly broad range of weights, however, with no firm basis for preferring one calculation over another. Values of parameters and their associated standard deviations (based on random error theory) proved insensitive to the relative weights within the indicated range of acceptable residuals. Fits to the molecular intensity curve were slightly less stable.

In contrast, the correlation coefficients, $\rho_{i j}{ }^{*}$, connecting the various geometrical parameters changed markedly with changes in the weight of microwave relative to diffraction data. The $\rho_{i j}$ 's are related to the functional dependence of the observables on the parameters, but are independent of the actual values of $I_{0}(s)$ or $I_{b}$, for example, and are only slightly dependent on the reference geometry at which they are evaluated. Their variation with changes in relative weights for the two experiments is the most important result of the mixing of the two sets of data, as discussed below.

Although the nonrandom nature of the errors precludes a rigorous statistical analysis of the uncertainties in the structure parameters derived from the combined analysis, a rough but qualitatively useful assessment can be made as follows. Let us formulate subjective error matrices $\mathbf{M}_{x, \text { ed }}$ and $\mathbf{M}_{x, m w}$ for the parameters $r_{\text {Xeo }}$, $r_{\mathrm{XeF}}$, and $\alpha$, corresponding to the electron diffraction and microwave studies, separately. The diagonal elements are taken to be the physically reasonable accumulated errors listed in Table 1, and the off-diagonal elements are generated with the correlation coefficients from the separate least-square fittings. It might be argued that the subjective $\sigma_{i}$ should be modified about the mean to make the regression slopes $\rho_{i j} \sigma_{i} \sigma_{j} / \sigma_{i}{ }^{2}$ simulate those of the least-squares fittings in those cases where $\rho_{i j}$ is large, but we chose not to do this. If the normal properties of error matrices are attributed to the subjective representations defined above, it follows that the error matrix corresponding to an optimum averaging of the electron diffraction and microwave data is the inverse of the matrix with elements $\left[\left(\mathbf{M}_{x, \text { ed }}{ }^{-1}\right)_{i j}+\left(M_{x, \mathrm{mw}}{ }^{-1}\right)_{i j}\right]$. Correlations between parameters are such that the implied standard errors are somewhat less than $\left(\sigma_{\text {ed }}{ }^{-2}+\sigma_{\mathrm{mw}}{ }^{-2}\right)^{-\frac{1}{2}}$. The standard errors thus calculated for the composite analysis are entered in column $B$ of Table 1 , treating the microwave uncertainties as if they were standard errors.

DISCUSSION

Because the equations relating diffraction and spectroscopic observations to molecular structure may contain systematic deficiencies which are larger than the

* See footnote a, Table 2.

J. Mol. Structure, 8 (1971) 383-394 
purely random errors of experiment, and because our understanding of their deficiencies is, at present, quite imperfect, there exists no rigorous method to transform the statistical data of least-squares analyses to physically significant standard errors*. Although the present case makes this shortcoming particularly obvious, similar troubles are probably the rule rather than the exception in most current, carefully executed electron diffraction studies. A common tendency in the literature is to treat residuals in intensity as if they were statistically distributed and to neglect systematic effects. This neglect can lead to absurdly low derived uncertainties, particularly when two close internuclear distances are being resolved. One scheme of aid in assessing plausible heavy atom-light atom uncertainties, as discussed above, is to utilize a Fourier transform to render more apparent the nonrandom residuals. Other schemes have been suggested elsewhere ${ }^{\sigma}$.

Short of a rigorous method for eliminating systematic discrepancies between theory and experiment, the best method for improving the reliability of mutually interfering (i.e., correlated) parameters is to add to the analyses some independent observations with a different pattern of correlation. The increased confidence to be gained is far more important than the factor $(n-m)^{\frac{1}{2}}$ characteristic of the mere increase in the number of observations being fitted. In the present study, as can be seen from Table 2. it turned out to be possible to mitigate the excessive correlation between $r_{\mathbf{X e O}}$ and $\alpha$ inherent in the microwave data and, simultaneously, to moderate the coupling between $r_{\mathrm{XeO}}$ and $r_{\mathrm{XeF}}$ engendered by the diffraction data, simply by mixing the two sets of data with appropriate weights. Thus, the weak points of each individual analysis could be remedied by the information from the other. If valid statistical analyses had been available, the standard deviations of the parameters themselves would have been adequate indicators of the effectiveness of the combined analysis. In the absence of such analyses the correlation coefficients play a more significant, if still subjective role.

That the electron diffraction structure, for which the proper resolution of $r_{\mathrm{XeO}}$ is so sensitive to failures in scattering theory, should agree so well with the microwave structure, with its poorly located xenon atom, is a pleasing result that could not have been foreseen. We may conclude, then, that for $\mathrm{XeOF}_{4}$, the present composite analysis effectively removes the objections to the structure results derived from either experiment alone.

\section{ACKNOWLEDGMENTS}

We are deeply indebted to Dr. Henry Selig for donating the sample of $\mathrm{XeOF}_{4}$.

\footnotetext{
* Standard deviations computed from the conventional $B^{-1}$ matrix elements using the usual formula have little or no statistical significance. We include them in Table 1 (the values in parentheses) merely to emphasize the disparity between them and more reasonable estimates of parameter uncertainties based on other considerations.
} 
It is a pleasure to acknowledge a generous allowance of computing time from the Michigan Computing Center.

\section{REFERENCES}

1 See for example K. Kuchitsu and S. Konaka, J. Chem. Phys., 45 (1966) 4342, and references therein.

2 K. Kuchiţsu, J. Chem. Phys., 49 (1968) 4456; K. Kuchitsu, T. Furuyama and Y. Morino, J. Mol. Siructure, 4 (1969) 41.

3 J. F. Martins AND E. B. Wilson, JR., J. Chem. Phys., 41 (1964) 570.

4 J. F. MARTINS AND E. B. WILSON, $J_{R}$, have since published several sets of structural parameters derived from their rotational constants using variants of the substitution method. See $J$. Mol. Spectry., 26 (1968) 410.

5 F. B. Clippard, Doctoral Dissertation, The University of Michigan, 1969.

6 L. S. BARTEll, in A. Weissberger AND B. W. Rossiter (Editors), Physical Methods in Chemistry, 4th Ed., Interscience, New York, in press.

7 R. M. Gayin, JR. ANd L. S. Bartelt, J. Chem. Phys., 48 (1968) 2460.

8 H. L. CoX, JR., Doctoral Dissertation, Indiana University, 1967.

9 C. Tavard, D. Nicolas and M. Rouault, J. Chim. Phys., 64 (1967) 540.

10 R. F. Pohler and H. P. Hanson, J. Chem. Phys., 42 (1965) 2347.

11 W. Heisenderg, Phys. Z., 32 (1931) 737.

12 G. HAGEN, Acta Chem. Scand., 21 (1967) 465.

13 S. J. Crvin, Molecular Vibrations and Mean Square Amplitudes, Elsevier, Amsterdam, 1968, p. 325. Calculations performed by J. BrunvolL.

14 Subsequent calculations by S. J. Cyvin, J. Brunvolt and A. G. Robietre, J. Mol. Structure, 3 (1969) 259, however, support these estimates.

15 K. Venkateswarlu and K. B. Joseph, Acta Phys. Hung., 24 (1968) 139.

16 P. H. ClipPard, private communication, 1969.

17 D. F. Smith, Science, 140 (1963) 899; G. M. Begun, W. H. Fletcher ANd D. F. SMith, J. Chem. Phys., 42 (1965) 2236.

18 H. H. ClaAssen, The Noble Gases, D. C. Heath and Co., Chicago, Ill., 1966.

19 E. J. Jacob and L. S. Bartell, J. Chem. Phys., 53 (1970) 2231.

J. Mol. Structure, 8 (1971) 383-394 